möglich. Beide Autoren schlagen die Änderung verschiedener rechtlicher Grundlagen vor. Im Fall der Streichung staatlicher Zuschüsse käme dies jedoch einem Parteiverbot durch die Hintertür gleich.

Johannes Haas

\title{
Parteimitglieder - ertragreiche Studie für Praxis und Forschung
}

Spier, Tim, Markus Klein, Ulrich von Alemann, Hanna Hoffmann, Annika Laux, Alexandra Nonnenmacher und Katharina Rohrbach (Hrsg.): Parteimitglieder in Deutschland, VS Verlag für Sozialwissenschaften, Wiesbaden 2011, 213 Seiten, € 27,99.

Gut ein Jahrzehnt nach der - bis dato in ihrer bundesweiten Auslegung singulären - Potsdamer Parteimitgliederstudie 1998 liegt nun eine erste komprimierte Veröffentlichung wesentlicher Befunde ihrer jüngeren Replikation, der „Deutschen Parteimitgliederstudie 2009“, vor, die von einem Düsseldorfer Forscherteam um Markus Klein und Ulrich von Alemann, erstellt worden ist. Diese Nachfolgestudie, das sei vorweg festgestellt, steht ihrer vielzitierten Vorgängerarbeit, an deren Untersuchungsanlage und Fragebogenduktus sie sich sinnvollerweise weitgehend orientiert, an analytischer Klarheit und methodischer Solidität in nichts nach. Die forschungspraktische Entscheidung, das damals von Wilhelm P. Bürklin und seiner Potsdamer Forschungsgruppe entwickelte Umfragedesign fortzuschreiben, macht zweifellos Sinn, gerade weil, wie die Autoren im Vorwort schreiben, „die komplexen Hintergründe des Rückgangs und Wandels parteipolitischer Partizipation in Deutschland“ nicht nur in Expertenzirkeln der Parteienforschung, sondern auch einer breiteren politischen Öffentlichkeit gewärtig sind. Erst wenn identische Variablensets zugrunde gelegt werden, treten differente Entwicklungslinien im Zeithorizont der Mitgliedschaften der Parteien deutlich zutage.

Die empirische Basis der neuen Untersuchung ist breit: Befragt wurden rund 9.000 Parteimitglieder (das entspricht einer guten Rücklaufquote von 58 Prozent) und zusätzlich 2.400 Probanden im Rahmen einer repräsentativen Bevölkerungsumfrage. Bei letzterer entfällt je ein Drittel auf Nichtmitglieder, ehemalige Mitglieder und organisierte Parteimitglieder, die über das Parteienspektrum streuen. Ostdeutschland ist in der Stichprobe der schriftlichen Mitgliederbefragung überproportional vertreten, tatsächlich sind, gemessen am Bevölkerungsanteil (20 Prozent), Ostdeutsche in den Parteien mit zwölf Prozent unterrepräsentiert. Die Drittelung der Bevölkerungsumfrage sowie die gesonderte Auswertung jener Befragtenteilgruppen, die 1998 und 2009 der Partei ihrer Wahl kurz zuvor beigetreten waren, resultiert aus dem Vorhaben, die in der Literatur zum Parteienwandel erörterte These zu testen, derzufolge bei Neumitgliedern ein ressourcenstarker Typus dominiert, der den sozialstrukturellen Wandel der Parteien vorantreibt und auf dieser Ebene Parteienunterschiede tendenziell verwischt. Erkennbar wird hier, neben aller wohlbegründeten Kontinuität des Untersuchungskonzepts, ein eigenständiges, innovatives Element im Forschungsprogramm der Düsseldorfer Studie.

Das Buch gliedert sich in zwölf Einzelbeiträge. Einleitend beschreiben Markus Klein und Ulrich von Alemann zunächst allgemein die Funktionen von Parteien in der Demokratie und sodann, ergänzt um Tim Spier als dritten Mitautor, die Gründe, weshalb Parteien auch im modernen Parteienstaat auf - möglichst viele - Mitglieder nicht verzichten können. Bei der 
- plausiblen - Begründung der Linkage-Funktion politischer Parteien kommen sie, was verwundert, ohne expliziten Bezug auf die Kartellpartei-These aus. Im Übrigen umschiffen Klein und Alemann elegant etliche Klippen der „Krise des Parteienstaates“, indem sie „Fälle von Ämterpatronage, illegaler Parteienfinanzierung und Korruption“ zu system-atypischen „Regelverletzungen“ des Parteienstaates erklären (S. 14) - wogegen sich einwenden ließe, dass die genannten Ärgernisse durchaus in der Struktur und Handlungslogik von Parteienregimen wurzeln und eben deshalb immer wieder auftreten.

Die Reihe der datenbasierten Teilstudien eröffnet Markus Klein, der die Vertretung sozialer Gruppen in der Mitgliedschaft der Parteien beschreibt. Frauen sind, seit 1998 so gut wie unverändert, deutlich unterrepräsentiert, desgleichen Jüngere unter 34 Jahren, letztere jedoch mit seither rückläufiger Tendenz. Nur noch jedes vierte Parteimitglied hat einen Hauptschulabschluss; hier fällt der Rückgang stärker aus als im Durchschnitt der Bevölkerung. Der Arbeiteranteil bei erwerbstätigen Mitgliedern „ist nur knapp halb so hoch wie ihr Anteil in der erwerbstätigen Bevölkerung insgesamt" (S. 51). Der Befund bestätigt die landläufige Annahme einer zunehmenden Akademisierung der Mitgliederbasis. Und nachweisbar ist auch, dass Parteimitglieder heutzutage größere „partizipationsrelevante Ressourcen “ (S. 58), also Bildung und ein ausgeprägteres Bewusstsein eigener Einflusschancen („efficacy“) haben. Indessen zeigt ein Vergleich der Neumitglieder von 1998 und 2009, dass die Rekrutierungsmuster, ausweislich der bekannten sozialstrukturellen Variablen, über die Zeit hinweg „relativ stabil“ geblieben sind.

Annika Laux stellt in ihrem Beitrag, mit Bezug auf das erprobte Anreizmodell von Patrick Seyd und Paul Whiteley (1992), typische Beitrittsmotive von Parteimitgliedern vor. Als wichtigstes erweist sich das Gesinnungsmoment, das heißt der Vorsatz, sich für die Ziele der Partei einzusetzen und diese dadurch zu stärken. Bei neu eingetretenen Mitgliedern spielt auch die Erwartung, dass politische Arbeit Spaß macht, eine wesentliche Rolle. Daran anschließend untersucht Hanna Hoffmann individuelle Beitrittsgründe von Parteimitgliedern. Der feine, aber analytisch nachvollziehbare Unterschied von Motiven und Gründen lässt sich so verstehen, dass letztere etwas über die persönliche biographische Grundausstattung als zoon politikon aussagen: Es sind, so zeigen die Umfragedaten, insbesondere ein generelles politisches Interesse sowie eine hohe Selbstzuschreibung politischer Kompetenz, die einen Parteibeitritt befördern.

Tim Spier hat für seinen Beitrag das Aktivitätsniveau und -potential ausgewertet. Bemerkenswert ist, dass sich knapp drei Viertel aller Parteimitglieder als „vergleichsweise inaktiv“ outen (S. 99). Aktive finden sich überdurchschnittlich häufig unter Jüngeren, Angehörigen der mittleren und oberen Mittelschicht (gemäß Selbsteinstufung) und, mit Einschränkungen, auch unter höher Gebildeten. Und: „Zusammenfassend können wir sagen, dass insbesondere diejenigen in Parteien aktiv werden, die sich konkrete und direkte Vorteile davon versprechen" (S. 108). Das ist nun keineswegs anrüchig, sondern eine logische Folge der Rekrutierungsfunktion von Parteien für Ämter und Wahlmandate in einer Demokratie. Zwar stellen die „ämterorientierten Aktiven“ (22 Prozent) den harten Kern der Parteitätigkeit. Doch es gibt auch die „geselligkeitsorientierten Aktiven“, die vornehmlich im sozialen Leben ihrer Partei aufgehen. Diesem Typus lassen sich immerhin 14 Prozent der Mitglieder zuordnen.

Ebenfalls Tim Spier stellt anschließend die politischen Einstellungen von Parteimitgliedern dar. Gemessen an der Elle der Links-Rechts-Skala bewegen sich Deutsche mit Parteibuch insgesamt eher „zur politischen Mitte“ hin und besetzen dabei stets „im Vergleich zu ihrer Wählerschaft pointiertere Positionen“" (S. 131). Alexandra Nonnenmacher geht in ihrem 
Beitrag der Frage nach, wie zufrieden Parteimitglieder eigentlich mit ihrer jeweiligen Partei sind. Dass insgesamt nur 14 Prozent Unzufriedenheit bekunden (dabei relativ mehr in den Volksparteien CDU/CSU und SPD), mag angesichts des Staubs, der mit der Dauerbeschwörung von „Parteienverdrossenheit“ öffentlich aufgewirbelt wird, überraschen. Wenn Mitglieder mit ihrer Partei hadern, begründen sie dies am häufigsten mit kritisierten Sachentscheidungen in Bund, Land oder Gemeinde.

Was wünschen sich Mitglieder von ihren Parteien? Annika Laux zeigt im Spiegel der Daten Folgendes auf: Gewünscht werden ganz überwiegend Geschlossenheit, auch im direkten Präferenzvergleich mit innerparteilicher Diskussion, ferner der Nachweis von Problemlösungskompetenz, die - allerdings hier weniger eindeutig - der Betonung programmatischer Grundwerte vorgezogen wird. Für Parteimitglieder hat „Responsivität“ offensichtlich auch Grenzen: Dem Votum der Mitglieder soll vor der Wählermeinung Vorrang eingeräumt werden. Für eine Urwahl von Parteivorstand und Bundestagskandidaten sowie für einen innerparteilichen Direktentscheid bei Sachfragen optieren wiederum deutliche Mitgliedermehrheiten in allen Parteien.

Ähnlich den Nichtwählern zählen auch Personen, die aus Parteien ausgetreten sind, zu weithin unbekannten Wesen, da sie mit Umfragen gemeinhin schwer erfassbar sind. Wie Katharina Rohrbach in ihrem Beitrag ausführt, unterscheidet die Parteimitgliederstudie 2009 nach den drei Untergruppen der Stammmitglieder, der Austrittsgefährdeten und der ehemaligen Mitglieder. Letztere werden mithilfe der Bevölkerungsstichprobe statistisch erfasst. Als austrittsgefährdet waren zum Zeitpunkt der Erhebung insgesamt zwölf Prozent der Mitglieder anzusehen, typischerweise solche, die inaktiv sind, der Partei schon länger angehören und sich über Sachentscheidungen geärgert haben. Ein dominantes Motiv für tatsächlichen Austritt ist vor allem das Gefühl, selbst nichts bewirken zu können.

Im abschließenden Beitrag skizzieren Markus Klein und Tim Spier die Zukunftsperspektiven innerparteilichen Engagements: Wird infolge des grassierenden Mitgliederschwunds die Funktionsfähigkeit der Parteien gefährdet? Einerseits werten es die Autoren als „misslich, dass Frauen, Niedriggebildete und Menschen mit einfachem sozialen Hintergrund unter den deutschen Parteimitgliedern unterrepräsentiert sind“ (S. 205). Andererseits stimmt zuversichtlich, dass die Zahl der Aktiven innerhalb der Parteien zunimmt. Um die Verbindung zwischen Parteien und Bevölkerung (wieder) zu stärken, lautet die Empfehlung, die Bildungsanstrengungen generell zu intensivieren und innerparteiliche Abläufe und Routinen offener zu gestalten.

Der vorliegende Sammelband ist ein Gewinn gleichermaßen für die Parteienforschung wie für die politische Praxis. Alle Beiträge sind erklärtermaßen für ein breiteres Publikum geschrieben und kommen daher, was die analytische Präzision keineswegs schmälert, fast gänzlich ohne Fußnoten aus. Die Beiträge überzeugen ausnahmslos sowohl durch die Dichte des präsentierten empirischen Materials als auch durch ein ausgewogenes Urteil. Als nachteilig kann angesehen werden, dass auf eine nach Ostdeutschland und Westdeutschland differenzierte Auswertung der Daten konsequent verzichtet worden ist. Es wäre nämlich aufschlussreich zu erfahren, ob sich der mit anderen Umfragedaten belegte Langzeittrend einer Konvergenz ost- und westdeutscher Einstellungen auch auf der Ebene der Parteimitglieder abbildet. In dieser Hinsicht darf man auf ergänzende Publikationen der Düsseldorfer Forschergruppe gespannt sein. 